\title{
Performance analysis of the MAP equalizer within an iterative receiver including a channel estimator
}

\author{
Noura Sellami \\ ISECS \\ Route Menzel Chaker km 0.5 \\ B.P 868, 3018 Sfax, Tunisia
}

\author{
Aline Roumy \\ IRISA-INRIA \\ Campus de Beaulieu \\ 35042 Rennes Cedex, France
}

\author{
Inbar Fijalkow \\ Laboratoire ETIS, UMR 8051 \\ ENSEA-UCP-CNRS , 6 av. du Ponceau \\ 95014 Cergy-Pontoise, France
}

\begin{abstract}
In this paper, we consider an iterative receiver composed of a Maximum A Posteriori (MAP) equalizer and a MAP decoder. During the iterations, the equalizer and the decoder exchange extrinsic information and use them as a priori in order to improve their performance. We consider here an iterative receiver including a channel estimator. We propose to study analytically the impact of both the $a$ priori information and the channel estimation errors on the equalizer performance. We show that it is equivalent to a shift in terms of signal-to-noise ratio (SNR) and we provide an analytical expression. Simulation results show that the analytical expression we give approximate quite well the equalizer performance.
\end{abstract}

\section{INTRODUCTION}

The optimal receiver for a frequency selective coded channel performs joint equalization and decoding which makes its complexity generally prohibitive. A solution achieving a good complexity/performance trade-off is to use an iterative receiver composed of a soft-input soft-output (SISO) equalizer and a SISO decoder [1]. The basic idea behind iterative processing is to exchange extrinsic information among the equalizer and the decoder in order to achieve successively refined performance. The optimal SISO algorithm, in the sense of minimum bit error rate (BER), to be used for equalization and decoding is the symbol MAP algorithm [2]. Hence, in this paper, we consider an iterative receiver composed of a MAP equalizer and a MAP decoder. We propose to study analytically the impact on the equalizer performance of both the channel estimation errors and the a priori information provided by the channel decoder. To do that, we follow the approach of [3] and [4] which studied the impact of channel estimation errors on the equalizer performance. In [3], Gorokhov studied the impact of channel estimation errors on the performance of the Viterbi equalizer and showed that it is equivalent to a loss in SNR and evaluated this loss. In [4], we have extended the study to a List-type MAP equalizer prefiltered by the whitened matched filter, in the case of multiple-input multiple-output (MIMO) systems.

In [5], we assumed that the channel is perfectly estimated by the receiver and we showed that the use of the a priori information by the MAP equalizer is equivalent to a gain in SNR. In this paper, we consider a more realistic scheme where the channel estimation is not perfect. In this case, we will show that the use of both the a priori information and the channel estimate by the MAP equalizer is equivalent to a shift in SNR and we will give a closed form of this shift.

This work is a first step in the study of the convergence analysis of iterative receivers. Most analyses are based on extrinsic information transfer (EXIT) charts [6]. These analyses use generally simulations since it is difficult to study analytically the performance of a MAP equalizer having a large number of states. Actually, analytical studies based on the EXIT function have been performed when the trellis has only two states [7]. The contribution of our paper is to give an analytical study of the MAP equalizer performance when the number of states is greater than two.

Throughout this paper scalars and matrices are lower and upper case respectively and vectors are underlined lower case. $(.)^{T}$ denotes the transposition and $I_{m}$ is the $m \times m$ identity matrix.

\section{SYSTEM MODEL}

We consider a coded data transmission system over a frequency selective channel depicted in Figure 1. The input information bit sequence is first encoded with a convolutional encoder. The output of the encoder is interleaved, mapped to the symbol alphabet $\mathcal{A}$. For simplicity, we will consider only the BPSK modulation $(\mathcal{A}=\{+1,-1\})$. We assume that transmissions are organized into bursts of $T$ symbols. The channel is supposed to be invariant during one burst. The received baseband signal sampled at the symbol rate at time $k$ is

$$
x_{k}=\sum_{l=0}^{L-1} h_{l} s_{k-l}+n_{k}
$$

where $L$ is the channel memory. In this expression, $n_{k}$ are modeled as independent samples of a real white Gaussian noise with normal probability density function (pdf) $\mathcal{N}\left(0, \sigma^{2}\right)$ where $\mathcal{N}\left(\alpha, \sigma^{2}\right)$ denotes a Gaussian distribution with mean $\alpha$ and variance $\sigma^{2}$. The term $h_{l}$ is the $l^{\text {th }}$ tap gain of the channel, which is assumed to be real valued. Let $\underline{s}=\left(s_{T-1}, \ldots, s_{1-L}\right)^{T}$ be the $(L+T-1)$-long vector of coded symbols and $\underline{n}=$ $\left(n_{T-1}, \ldots, n_{0}\right)^{T}$ be the $T$-long noise vector. The output of the channel is the $T$-long vector $\underline{x}=\left(x_{T-1}, \ldots, x_{0}\right)^{T}$ defined as

$$
\underline{x}=\tau(h) \underline{s}+\underline{n}
$$


where $\tau(h)$ is a $T \times(T+L-1)$ Toeplitz matrix with its first row being $\left(h_{0}, h_{1}, \ldots, h_{L-1}, 0, \ldots, 0\right)$ and its first column $\left(h_{0}, 0, \ldots, 0\right)^{T}$.

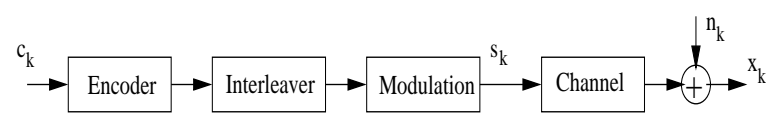

Fig. 1. Transmitter structure

When the channel is known and no a priori information is provided to the equalizer, the data estimate according to the sequence MAP criterion (equivalently the maximum likelihood (ML) criterion since there is no a priori) is given by

$$
\underline{\hat{s}}_{M A P}=\arg \min _{\underline{u}}\left(\|\underline{x}-\tau(h) \underline{u}\|: \underline{u} \in \mathcal{A}^{T+L-1}\right) .
$$

Now, we consider a particular error event characterized by its length $m$ [8]. Thus, we suppose that there exists an interval of size $m$ such that all the symbols of $\underline{\hat{s}}$ are different from the corresponding symbols of $\underline{s}$ while the preceding symbol and the following one are the same for $\underline{s}$ and $\underline{\hat{s}}$. Define $\underline{s}_{m}$ and $\underline{\hat{s}}_{m}$ to be the vectors of symbols corresponding to this interval and the vector of errors $\underline{e}_{m}=\underline{\hat{s}}_{m}-\underline{s}_{m}$. A subevent $\mathcal{E}_{m}$ of the error event is that $\underline{\hat{s}}_{m}$ is better than $\underline{s}_{m}$ in the sense of the ML metric

$$
\mathcal{E}_{m}:\left\|\underline{x}_{m}-\tau_{m}(h) \underline{\hat{s}}_{m}\right\| \leq\left\|\underline{x}_{m}-\tau_{m}(h) \underline{s}_{m}\right\|
$$

where $\underline{x}_{m}$ is the subvector of $\underline{x}$ and $\tau_{m}(h)$ is the block of $\tau(h)$ corresponding to the error interval. The probability $P\left(\mathcal{E}_{m}\right)$ of $\mathcal{E}_{m}$ is given by [8]:

$$
P\left(\mathcal{E}_{m}\right)=Q\left(\frac{\left\|\underline{\varepsilon}_{m}\right\|}{2 \sigma}\right)
$$

where $\underline{\varepsilon}_{m}=\tau_{m}(h) \underline{e}_{m}$ and $Q(\alpha)=\frac{1}{\sqrt{\pi}} \int_{\alpha}^{\infty} \exp \left(-y^{2}\right) d y$. Let $\Sigma_{m}$ be the set of all possible error events of length $m$. Then, the probability, $P\left(\Sigma_{m}\right)$, that any error event is of length $m$ is bounded by the sum of the probabilities of the subevents $\mathcal{E}_{m}$

$$
P\left(\Sigma_{m}\right) \leq \sum_{\mathcal{E}_{m}} P\left(\mathcal{E}_{m}\right)
$$

Let $d_{\text {min }}$ be the channel minimum distance [8]. Because of the exponential decrease of the Gaussian distribution function, the overall probability of error $P(\Sigma) \leq \sum_{m} P\left(\Sigma_{m}\right)$ will be dominated at high SNR by the term involving the minimum value $d_{\min }$ of $\left\|\underline{\varepsilon}_{m}\right\|$. Thus

$$
P(\Sigma) \simeq Q\left(\frac{d_{\min }}{2 \sigma}\right)
$$

Our goal is to find an approximation of $P(\Sigma)$ when the equalizer is integrated into an iterative receiver including a channel estimator.

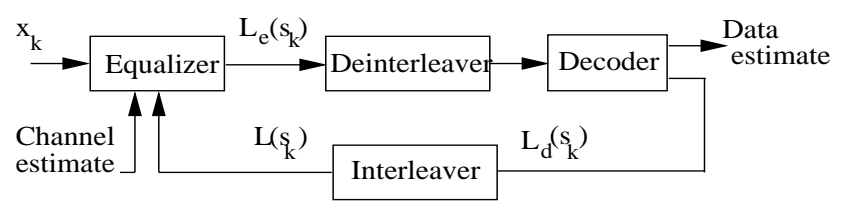

Fig. 2. Transmitter structure

\section{ITERATIVE RECEIVER}

As shown in Figure 2, the receiver consists of two soft-input soft-output (SISO) processors, the equalizer and the decoder. We consider only the MAP approach for both equalization and decoding, using the BCJR algorithm [2]. The MAP equalizer computes the a posteriori probabilities (APPs) on the coded bits, $P\left(s_{k}=s \mid \underline{x}, \underline{\underline{h}}\right), s \in \mathcal{A}, 1-L \leq k \leq T-1$, using the received vector $\underline{x}$ and the channel estimation vector $\underline{\tilde{h}}=\left(\tilde{h}_{0}, \cdots, \tilde{h}_{L-1}\right)^{T}$, and outputs the log-likelihood ratios (LLRs) [6]:

$$
\begin{aligned}
L_{e}\left(s_{k}\right) & =L\left(s_{k} \mid \underline{x}, \underline{\tilde{h}}\right)-L\left(s_{k}\right) \\
& =\log \frac{P\left(s_{k}=+1 \mid \underline{x}, \underline{\tilde{h}}\right)}{P\left(s_{k}=-1 \mid \underline{x}, \underline{\tilde{h}}\right)}-\log \frac{P\left(s_{k}=+1\right)}{P\left(s_{k}=-1\right)}
\end{aligned}
$$

which are the a posteriori LLRs $L\left(s_{k} \mid \underline{x}, \underline{\tilde{h}}\right)$ minus the a priori LLRs $L\left(s_{k}\right)$. These a priori LLRs are provided by the decoder. At the first receiver iteration, $L\left(s_{k}\right)=0$ since no a priori information is available. The LLRs $L_{e}\left(s_{k}\right)$ are then deinterleaved and provided to the decoder as input information, in order to refine its reliability. The MAP decoder computes the APPs $P\left(s_{k}=s \mid \underline{r}\right), \underline{r}=\left(L_{e}\left(s_{1-L}\right), \cdots, L_{e}\left(s_{T-1}\right)\right)^{T}$, and outputs the LLRs

$$
L_{d}\left(s_{k}\right)=\log \frac{P\left(s_{k}=+1 \mid \underline{r}\right)}{P\left(s_{k}=-1 \mid \underline{r}\right)}-\log \frac{P\left(s_{k}=+1\right)}{P\left(s_{k}=-1\right)} .
$$

These LLRs are then interleaved and provided to the equalizer as a priori, $L\left(s_{k}\right)$, at the next iteration. After some iterations, hard decisions are taken on the information bits by the decoder.

\section{PERFormance ANALYSis}

Now, we want to evaluate the impact of both the a priori information and the channel estimation errors on the MAP equalizer performance. The study will be done here for the equalizer using the sequence MAP criterion. It holds for the symbol MAP equalizer using the BCJR algorithm [2] since the two equalizers have almost the same performance as shown in [9, page 814].

Proposition: Suppose we are given a frequency selective channel with a memory of $L$ and an additive white Gaussian noise (AWGN) with noise variance $\sigma^{2}$. Assume that the outputs of an AWGN channel with noise variance $\sigma_{a}^{2}$ are also available as observations (corresponding here to the $a$ priori observations). Suppose that the channel is estimated by using a perfect training sequence of length $T_{0}$, having ideal 
autocorrelation properties. The estimates $\tilde{h}_{l}$ of the tap gains $h_{l}$, for $0 \leq l \leq L-1$, are thus modeled as $\tilde{h}_{l}=h_{l}+\sigma_{e} k_{l}$, where $k_{l}$ are independent Gaussian random variables with zero mean and variance 1 and $\sigma_{e}=\frac{\sigma}{\sqrt{T_{0}}}$.Then, at high SNR, the MAP equalizer using the a priori information and the channel estimate is equivalent to the MAP equalizer having no a priori information and a perfect channel knowledge but with an equivalent signal-to-noise ratio

$$
S \hat{N} R_{e s t}=S N R\left(1+\frac{8 \mu^{2}}{d_{\min }^{2}}\right)\left(1+\frac{L r^{2}}{1+\frac{8 \mu^{2}}{d_{\min }^{2}}}\right)^{-1}
$$

where $S N R$ is the true signal-to-noise ratio, $\mu=\frac{\sigma}{\sigma_{a}}$ and $r=\frac{\sigma_{e}}{\sigma}$.

Remark: The representation of the a priori information as the outputs of an AWGN channel is a good approximation of the decoder outputs. Actually, it has been shown in [11][10][6] that it is equivalent to have at the equalizer input a set of observations

$$
z_{k}=s_{k}+w_{k}
$$

where $w_{k} \sim \mathcal{N}\left(0, \sigma_{a}^{2}\right)$. Thus, the LLRs $L\left(s_{k}\right)$ fed back from the decoder can be modeled as independent and identically distributed (i.i.d) samples from a random variable with the conditional pdf $\mathcal{N}\left(\frac{2 s_{k}}{\sigma_{a}^{2}}, \frac{4}{\sigma_{a}^{2}}\right)$ for some $\sigma_{a}^{2}[10][6]$.

\section{Proof:}

The proof is divided into three parts. First, the probability of an error subevent of length $m, P\left(\mathcal{E}_{m}\right)$, is derived and then upper bounded. Finally, the overall probability of error, $P(\Sigma)$, is calculated in order to find an approximation of the equivalent SNR.

Proof-part1: $P\left(\mathcal{E}_{m}\right)$

Let $\underline{h}=\left(h_{0}, \cdots, h_{L-1}\right)^{T}$ be the vector of true channel parameters and $\underline{\tilde{h}}=\left(\tilde{h}_{0}, \cdots, \tilde{h}_{L-1}\right)^{T}$ be its estimate. Taking into account the a priori information and the channel estimation errors, the a posteriori probability of the sequence $\underline{s}$ is given by

$$
p(\underline{s} \mid \underline{x}, \underline{z}, \underline{\tilde{h}}) \propto \exp \left(-\frac{\|\underline{x}-\tau(\tilde{h}) \underline{s}\|^{2}}{2 \sigma^{2}}\right) \exp \left(-\frac{\|\underline{z}-\underline{s}\|^{2}}{2 \sigma_{a}^{2}}\right)
$$

where $\quad \underline{z}=\left(z_{T-1}, \ldots, z_{1-L}\right)^{T}$ and $\tau(\tilde{h})$ is a $T \times(T+L-1)$ Toeplitz matrix with its first row being $\left(\tilde{h}_{0}, \tilde{h}_{1}, \ldots, \tilde{h}_{L-1}, 0, \ldots, 0\right)$ and its first column $\left(\tilde{h}_{0}, 0, \ldots, 0\right)^{T}$. The data estimate according to the sequence MAP criterion is then given by

$\underline{\hat{s}}_{M A P}=\arg \min _{\underline{u}}\left(\|\underline{x}-\tau(\tilde{h}) \underline{u}\|^{2}+\frac{\sigma^{2}}{\sigma_{a}^{2}}\|\underline{z}-\underline{u}\|^{2}: \underline{u} \in \mathcal{A}^{T+L-1}\right) \quad\left\|\underline{\varepsilon}_{m}\right\|^{2}+4 m \mu^{2} \leq 2\left(\underline{E}_{m}^{T} \underline{b}_{m}-\underline{E}_{m}^{T} M_{m}(\Delta h) \underline{s}_{m}\right)$
A subevent $\mathcal{E}_{m}$ of the error event of length $m$ is that $\underline{\hat{s}}_{m}$ is better than $\underline{s}_{m}$ in the sense of the sequence MAP metric

$$
\begin{gathered}
\mathcal{E}_{m}:\left\|\underline{x}_{m}-\tau_{m}(\tilde{h}) \underline{\hat{s}}_{m}\right\|^{2}+\frac{\sigma^{2}}{\sigma_{a}^{2}}\left\|\underline{z}_{m}-\underline{\hat{s}}_{m}\right\|^{2} \leq \\
\\
\left\|\underline{x}_{m}-\tau_{m}(\tilde{h}) \underline{s}_{m}\right\|^{2}+\frac{\sigma^{2}}{\sigma_{a}^{2}}\left\|\underline{z}_{m}-\underline{s}_{m}\right\|^{2}
\end{gathered}
$$

where $\underline{z}_{m}$ is the subvector of $\underline{z}$ and $\tau_{m}(\tilde{h})$ is the block of $\tau(\tilde{h})$ corresponding to the error interval.

Let $\mu=\frac{\sigma}{\sigma_{a}}, \underline{y}=\left(x_{T-1}, x_{T-2}, \cdots, x_{0}, \mu z_{T-1}, \cdots, \mu z_{1-L}\right)^{T}$, $M=\left((\tau(h))^{T}, \mu I_{T+L-1}\right)^{T}$ a $(L-1) \times(2 T+L-1)$ matrix and $\underline{b}=\left(n_{T-1}, n_{T-2}, \cdots, n_{0}, \mu w_{T-1}, \cdots, \mu w_{1-L}\right)^{T}$. Using (1) and (10), we can write

$$
\underline{y}=M \underline{s}+\underline{b} .
$$

Using $\underline{\tilde{h}}$ instead of $\underline{h}$, the data estimate according to the sequence MAP criterion is given by,

$$
\underline{\hat{s}}_{M A P}=\arg \min _{\underline{u}}\left(\|\underline{y}-\tilde{M} \underline{u}\|^{2}: \underline{u} \in \mathcal{A}^{T+L-1}\right)
$$

where $\tilde{M}=\left((\tau(\tilde{h}))^{T}, \mu I_{T+L-1}\right)^{T}$. Hence, (13) is equivalent to

$$
\mathcal{E}_{m}:\left\|\underline{y}_{m}-\tilde{M}_{m} \underline{\hat{s}}_{m}\right\|^{2} \leq\left\|\underline{y}_{m}-\tilde{M}_{m} \underline{s}_{m}\right\|^{2}
$$

where $\underline{y}_{m}$ is the $(2 m+L-1) \times 1$ subvector of $\underline{y}$ corresponding to the error interval and $\tilde{M}_{m}=\left(\left(\tau_{m}(\tilde{h})\right)^{T}, \mu I_{m}\right)^{T}$.

Let $\underline{e}_{m}=\underline{\hat{s}}_{m}-\underline{s}_{m}$, then (16) is equivalent to the event

$$
\left\|\tilde{M}_{m} \underline{e}_{m}\right\|^{2} \leq 2\left(\underline{e}_{m}^{T} \tilde{M}_{m}^{T}\left(\underline{y}_{m}-\tilde{M}_{m} \underline{s}_{m}\right)\right) .
$$

Let $M_{m}(\Delta h)=\tilde{M}_{m}-M_{m}$ and $\underline{b}_{m}=\underline{y}_{m}-M_{m} \underline{s}_{m}$, then we obtain

$$
\left\|\tilde{M}_{m} \underline{e}_{m}\right\|^{2} \leq 2\left(-\underline{e}_{m}^{T} \tilde{M}_{m}^{T} M_{m}(\Delta h) \underline{s}_{m}+\underline{e}_{m}^{T} \tilde{M}_{m}^{T} \underline{b}_{m}\right) .
$$

Using the assumptions given in [3], we obtain that $\left\|\tau_{m}(\tilde{h}) \underline{e}_{m}\right\| \longrightarrow\left\|\underline{\varepsilon}_{m}\right\|\left(1+\xi_{T_{0}}\right)$ where $\xi_{T_{0}}$ tends in probability to 0 , hence

$$
\begin{aligned}
\left\|\tilde{M}_{m} \underline{e}_{m}\right\|^{2} & =\left\|\tau_{m}(\tilde{h}) \underline{e}_{m}\right\|^{2}+4 m \mu^{2} \\
& \rightarrow\left\|\underline{\varepsilon}_{m}\right\|^{2}+4 m \mu^{2} .
\end{aligned}
$$

Replacing $\left\|\tilde{M}_{m} \underline{e}_{m}\right\|^{2}$ by $\left\|\underline{\varepsilon}_{m}\right\|^{2}+4 m \mu^{2}$ and $\tilde{M}_{m} \underline{e}_{m}$ by $E_{m}=$ $M_{m} \underline{e}_{m}$ in (18), leads to 


$$
\underline{E}_{m}^{T} M_{m}(\Delta h) \underline{s}_{m}=\underline{\varepsilon}_{m}^{T} M_{m}(\Delta h) \underline{s}_{m} .
$$

We suppose that $\Delta h=\underline{\tilde{h}}-\underline{h} \sim \mathcal{N}(0, C), C$ being the covariance matrix of $\Delta h$. Defining $C_{m}(s)=H_{L}\left(\underline{s}_{m}\right) C H_{L}\left(\underline{s}_{m}\right)^{T}$, $H_{L}\left(\underline{s}_{m}\right)$ being the Hankel matrix such as $H_{L}\left(\underline{s}_{m}\right) \Delta h=$ $M_{m}(\Delta h) \underline{s}_{m}$, we obtain

$$
\left\|\underline{\varepsilon}_{m}\right\|^{2}+4 m \mu^{2} \leq \chi_{s}
$$

where $\chi_{s} \sim \mathcal{N}\left(0, \Delta_{s}\right)$ with

$$
\begin{aligned}
\Delta_{s} & =4 \sigma^{2}\left\|\underline{E}_{m}\right\|^{2}+4 \underline{\varepsilon}_{m}^{T} C_{m}(s) \underline{\varepsilon}_{m} \\
& =4 \sigma^{2}\left\|\underline{E}_{m}\right\|^{2}\left(1+\frac{1}{1+\frac{4 m \mu^{2}}{\left\|\underline{\varepsilon}_{m}\right\|^{2}}} \frac{\underline{\varepsilon}_{m}^{T} C_{m}(s) \underline{\varepsilon}_{m}}{\sigma^{2}\left\|\underline{\varepsilon}_{m}\right\|^{2}}\right)
\end{aligned}
$$

Hence, the probability of the error event $P\left(\mathcal{E}_{m}\right)$ is given by

$$
\begin{aligned}
& P\left(\mathcal{E}_{m}\right)=Q\left(\frac{\left\|\underline{E}_{m}\right\|}{2 \sigma}\left(1+\frac{1}{1+\frac{4 m \mu^{2}}{\left\|\underline{\varepsilon}_{m}\right\|^{2}}} \frac{\underline{\varepsilon}_{m}^{T} C_{m}(s) \underline{\varepsilon}_{m}}{\sigma^{2}}\right)^{-1 / 2} \underline{\varepsilon}_{m} \|^{2}\right. \\
& =Q\left(\frac{\left\|\underline{\varepsilon}_{m}\right\|}{2 \sigma} \sqrt{1+\frac{4 m \mu^{2}}{\left\|\underline{\varepsilon}_{m}\right\|^{2}}}\left(1+\frac{1}{1+\frac{4 m \mu^{2}}{\left\|\underline{\varepsilon}_{m}\right\|^{2}}} \frac{\underline{\varepsilon}_{m}^{T} C_{m}(s) \underline{\varepsilon}_{m}}{\sigma^{2}\left\|\underline{\varepsilon}_{m}\right\|^{2}}\right)^{-1 / 2}\right.
\end{aligned}
$$

We suppose here that a perfect training sequence of length $T_{0}$ is used and then $\tilde{h}_{l}=h_{l}+\sigma_{e} k_{l}$, where $k_{l}$ are modeled as independent complex Gaussian random variables with zero mean and variance 1 and $\sigma_{e}=\frac{\sigma}{\sqrt{T_{0}}}$. Thus, $C_{m}(s) \rightarrow L \sigma_{e}^{2} I_{m}$, and $\underline{\varepsilon}_{m}^{T} C_{m}(s) \underline{\varepsilon}_{m} \rightarrow L \sigma_{e}^{2}\left\|\underline{\varepsilon}_{m}\right\|^{2}$. This leads to

$$
\begin{aligned}
& P\left(\mathcal{E}_{m}\right)=Q\left(\frac{\left\|\underline{\tilde{E}}_{m}\right\|}{2 \sigma}\right) \\
& =Q\left(\frac{\sqrt{\left\|\underline{\varepsilon}_{m}\right\|^{2}+4 m \mu^{2}}}{2 \sigma}\left(1+\frac{1}{1+\frac{4 m \mu^{2}}{\left\|\underline{\varepsilon}_{m}\right\|^{2}}} L r^{2}\right)^{-1 / 2}\right)
\end{aligned}
$$

where $r=\frac{\sigma_{e}}{\sigma}$.

Proof-part2: upper bound for $P\left(\mathcal{E}_{m}\right)$ :

In order to find an approximation of $P(\Sigma)$, the overall probability of error, we now want to find a lower bound for the quantity $\left\|\underline{\tilde{E}}_{m}\right\|$. Actually, at high SNR, this term will dominate the sum of the probabilities of the error events (because of the exponential decrease of the Gaussian distribution function). Let us consider first the case of perfect channel knowledge. By definition, $\left\|\underline{\varepsilon}_{m}\right\|^{2} \geq d_{\min }^{2}$. Moreover, we have $m \geq 2$. Thus, a lower bound for $\left\|\tilde{E}_{m}\right\|=\sqrt{\left\|\underline{\varepsilon}_{m}\right\|^{2}+4 m \mu^{2}}$ is given by

$$
\operatorname{bound}\left(\mu^{2}\right)=\sqrt{d_{\min }^{2}+8 \mu^{2}} .
$$

In [5], we showed that this bound is tight.

When the channel is not perfectly estimated, the probability of the error event can be rewritten as

$$
P\left(\mathcal{E}_{m}\right)=Q\left(\frac{\left\|\underline{\varepsilon}_{m}\right\|^{2}+4 m \mu^{2}}{2 \sigma \sqrt{\left\|\underline{\varepsilon}_{m}\right\|^{2} L r^{2}+\left\|\underline{\varepsilon}_{m}\right\|^{2}+4 m \mu^{2}}}\right) .
$$

We assume here that $L<<T_{0}$ which is equivalent to $L r^{2}<<1$. In this case, $\left\|\underline{\varepsilon}_{m}\right\|^{2} L r^{2}$ is negligible compared to $\left\|\underline{\varepsilon}_{m}\right\|^{2}$. When the error sequence allowing to attain the minimum distance is of length $m=2$, we can consider that the quantity obtained by calculating $\left\|\underline{\tilde{E}}_{m}\right\|$ taking $m=2$ and $\left\|\underline{\varepsilon}_{m}\right\|^{2}=d_{\min }^{2}$ is a lower bound for $\left\|\underline{\tilde{E}}_{m}\right\|$. Otherwise, an exhaustive search has to be done to find the lower bound.

\section{Proof-part3: $P(\Sigma)$}

As in the case without $a$ priori and perfect channel knowledge, at high SNR, the overall probability of error $P(\Sigma)$ can be approximated by

$$
P(\Sigma) \simeq Q\left(\frac{d_{\min }}{2 \sigma} \sqrt{1+\frac{8 \mu^{2}}{d_{\min }^{2}}}\left(1+\frac{1}{1+\frac{8 \mu^{2}}{d_{\min }^{2}}} L r^{2}\right)^{-1 / 2}\right) .
$$

Thus, the expression of the error probability given in (28) can be seen as the one given in (7) with an equivalent signal-tonoise ratio

$$
S \hat{N} R_{e s t}=S N R\left(1+\frac{8 \mu^{2}}{d_{\min }^{2}}\right)\left(1+\frac{L r^{2}}{1+\frac{8 \mu^{2}}{d_{\min }^{2}}}\right)^{-1} .
$$

\section{Simulation RESUlts}

In our simulations, we consider the following channels:

- Channel3: $(0.5 ; 0.71 ; 0.5)$

- Channel5: $(0.29 ; 0.50 ; 0.58 ; 0.50 ; 0.29)$

The modulation used is the BPSK. The transmissions are organized into bursts of 512 symbols. Figures 3 and 4 show the Bit Error Rate $(B E R)$ curves with respect to the SNR, for different values of the ratio $\mu=\frac{\sigma}{\sigma_{a}}$ and for $r=\frac{\sigma_{e}}{\sigma}=0.3$. This value of the ratio $r$ corresponds to an ideal training sequence of length $T_{0}=11$. Each curve is obtained while the ratio $\mu$ is kept constant. The solid lines indicate the receiver performance obtained by simulations. The dotted lines are obtained by shifting the curve corresponding to the case with no a priori and with a perfect channel knowledge $(\mu=0, r=0)$ by the values of the SNR shift: $10 \log _{10}(1+$ $\left.\frac{8 \mu^{2}}{d_{\min }^{2}}\right)-10 \log _{10}\left(1+\frac{L r^{2}}{1+\frac{8 \mu^{2}}{d_{\min }^{2}}}\right)$. Table.1 shows the values of the minimum error distance $d_{\min }$ and the minimum distance input error sequence for the channels of interest [12]. 


\begin{tabular}{|l|l|l|}
\hline & Channel3 & Channel5 \\
\hline$d_{\min }$ & 1.5308 & 1.0532 \\
\hline Error sequence & $(2,-2)$ & $(2,-2)$ \\
\hline \multicolumn{2}{|c}{ Table.1 }
\end{tabular}

For Channel3, we notice that the theoretical curves (dotted lines) approximate well the $B E R$. However, for larger $\mu$ $(\mu=0.83)$, the approximation becomes slightly erroneous. Moreover, Figure 4 shows that the approximation is better for Channel5. We can conclude that the approximation holds in general for $\sigma<\sigma_{a}$ and that it is better for Channel5.

Now, we propose to test the reliability of the term due to the channel estimation errors in (9). In Figure 5, the solid curves indicate the performance of the equalizer fed by the a priori when the channel is estimated $(r=0.3)$, for different values of $\mu$. The dotted curves are obtained by shifting the curves given by simulations for different values of $\mu$ and $r=0$ by the values of the SNR loss: $-10 \log _{10}\left(1+\frac{L r^{2}}{1+\frac{8 \mu^{2}}{d_{\min }^{2}}}\right)$. We notice that the dotted curves approximate well the solid ones independently of the values of $\mu$. Hence, the gap observed between the theoretical curves and the curves obtained by simulations in figures 3 and 4 is due to the term corresponding to the effect of the a priori and not to the term corresponding to the channel estimation errors in (9).

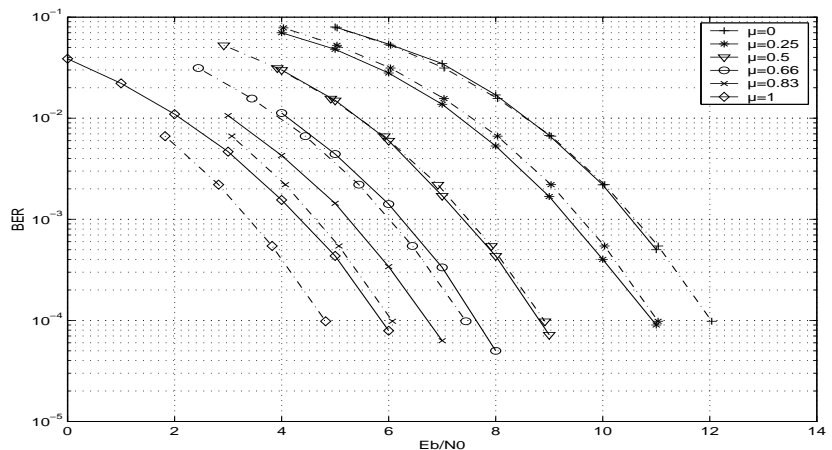

Fig. 3. Comparison of the equalizer performance and the theoretical performance when $r=\frac{\sigma_{e}}{\sigma}=0.3$, for different values of $\mu$, for Channel3.

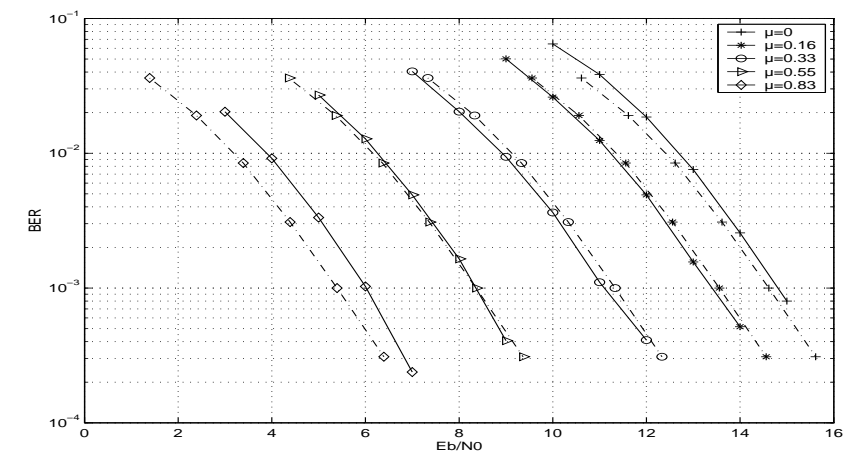

Fig. 4. Comparison of the equalizer performance and the theoretical performance when $r=\frac{\sigma_{e}}{\sigma}=0.3$, for different values of $\mu$, for Channel5.

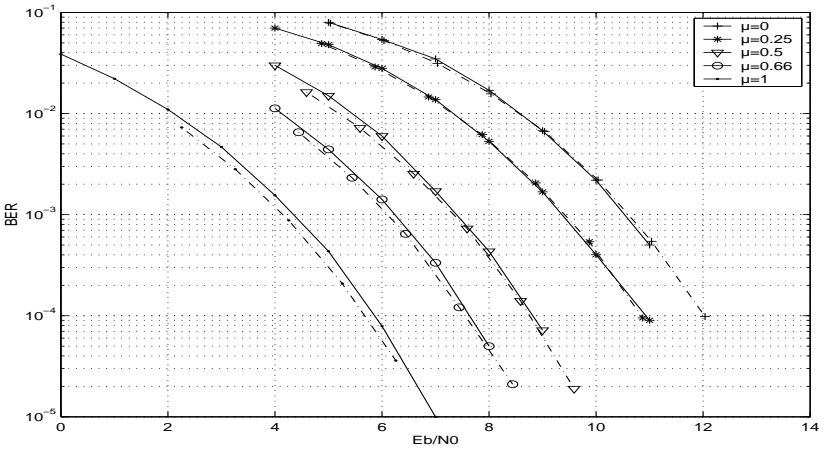

Fig. 5. The solid curves indicate the performance of the equalizer within the iterative receiver when the channel is estimated $(r=0.3)$. The dotted curves are obtained by shifting the curves given by simulations for different values of $\mu$ and $r=0$ by the values of the SNR loss.

\section{CONCLUSION}

In this paper, we considered an iterative receiver composed of a MAP equalizer and a MAP decoder. We proposed to study analytically the impact of both the a priori information and the channel estimation errors on the equalizer performance. We gave an approximation of the error probability which allows us to find an expression of the shift in terms of the SNR due to the use of the a priori information and the channel estimate. Simulation results showed that this expression gives a quite good approximation especially for long channels. This work is a first step in the study of the convergence analysis of iterative receivers.

\section{REFERENCES}

[1] C.Douillard, M.Jézéquel, C.Berrou, A.Picart, P.Didier, and A.Glavieux, "Iterative correction of intersymbol interference: turbo-equalization," European Trans. Telecommun., vol. 6, no. 5, pp. 507-511, 1995.

[2] L.R.Bahl, J.Cocke, F.Jelinek, and J.Raviv, "Optimal decoding of linear codes for minimizing symbol error rate," IEEE Trans. Inf. Theory, vol. IT-32, pp.284-287, March 1974.

[3] A.Gorokhov, "On the performance of the Viterbi equalizer in the presence of channel estimation errors," IEEE Signal Process. Letters, vol. 5, no. 12, pp. 321-324, December 1998.

[4] N.Sellami, I.Fijalkow, and S.Perreau, "Performance analysis of a Listtype equalizer over estimated MIMO frequency selective channels," EUSIPCO'02, Toulouse, France, September 2002.

[5] N.Sellami, A.Roumy, and I.Fijalkow, "On the analysis of the MAP equalizer performance within an iterative receiver," Signal Process. Advances in Wireless Comm. (SPAWC'04), Lisbon, Portugal, July 2004.

[6] M.Tüchler, R.Koetter, and A.Singer, "Turbo equalization: principles and new results," IEEE Trans. on Comm., vol. 50, no. 5, pp. 754-767, May 2002.

[7] A.Roumy, S.Guemghar, G.Caire, and S.Verdu, "Design methods for irregular repeat accumulate codes," IEEE Trans. on Inf. Theory, to appear August 2004.

[8] G.D.Forney, Jr., "Maximum-likelihood sequence estimation for digital sequences in the presence of intersymbol interference," IEEE Trans. Inf. Theory, vol. 18, pp. 363-378, May 1972.

[9] S. Benedetto and E. Biglieri, Principles of Digital Transmission with Wireless Applications, NewYork: Kluwer/Plenum, 1999.

[10] S.Ten Brink, "Convergence of iterative decoding," IEEE Electronic Letters, vol. 35, pp.806-808, May 1999.

[11] J.Hagenauer, E.Offer, and L. Papke, "Iterative decoding of binary block and convolutional codes," IEEE Trans. on Inf. Theory, vol. 42, Issue 2, pp. 429-445, March 1996.

[12] W.Ser, K.Tan, and K.Ho, "A new method for determining "unknown" worst-case channels for maximum-likelihood sequence estimation," IEEE Trans. on Comm., vol. 46, no. 2, pp. 164-168, February 1998. 